\title{
A geometric construction of solutions of the Toda lattice hierarchy
}

\author{
G.F. Helminck \\ University of Twente, Faculty of Applied Mathematics, P.O.Box 217, 7500 AE Enschede
}

\begin{abstract}
In this paper we present an analytic and geometric framework for the construction of solutions of the Toda lattice hierarchy.
\end{abstract}

Introduction. In [UT], Ueno and Takasaki introduced the Toda lattice hierarchy, a system of nonlinear differential difference equations. Their approach is of a formal character and does not consider convergence questions for the objects they are dealing with. Here we will describe a convergent setting in which one can construct solutions of the system mentioned above. A more detailed description of the different sections is as follows: the first section contains the algebraic formulation of the hierarchy in Lax-form and the reduction of the system to a set of equations for so-called "wavematrices". In the second section we give the geometric setting for the construction of the convergent solutions. The final section gives the construction of the wavematrices and the proof that they satisfy the equations discussed in the first section.

\section{$\S 1$ The equations.}

1.1 Since the Toda lattice hierarchy is defined as a system of differential equations for the matrixcoefficients of a number of $\mathbb{Z} \times \mathbb{Z}$-matrices, we first introduce some notations.

Let $R$ be a commutative ring. Then we write $M_{\mathbb{Z}}(R)$ for the $R$-module consisting of $\mathbb{Z} \times \mathbb{Z}$ matrices with coefficients from $R$. If $A=\left(A_{i j}\right)$ and $B=\left(B_{i j}\right)$ belong to $M_{\mathbb{Z}}(R)$, then the product $A \cdot B$ in $M_{\mathbb{Z}}(R)$, where

$$
(A \cdot B)_{i k}=\sum_{j \in \mathbb{Z}} A_{i j} B_{j k}
$$

is only defined for special $A$ and $B$. It always exists if $A$ or $B$ belongs to the diagonalmatrices

$$
\mathcal{D}(R)=\left\{A \mid A \in M_{\mathbb{Z}}(R), A_{i j}=0 \text { if } i \neq j\right\} .
$$

Moreover an element $A$ of $\mathcal{D}(R)$ is invertible if and only if all the $A_{i i}$ are invertible in $R$. In the sequel, an important role is played by the element $\Lambda$ of $M_{\mathbb{Z}}(R)$ given by

$$
\Lambda_{i j}=\left\{\begin{array}{l}
1 \text { if } j=i-1 \\
0 \text { if } j \neq i-1
\end{array}\right.
$$


It has an inverse $\Lambda^{-1}$ with entries given by

$$
\left(\Lambda^{-1}\right)_{i j}=\left\{\begin{array}{l}
1 \text { if } j=i+1 \\
0 \text { if } j \neq i+1 .
\end{array}\right.
$$

The matrix $\Lambda$ acts on $\mathcal{D}(R)$ by conjugation: $\left(\Lambda d \Lambda^{-1}\right)_{j j}=d_{j-1 j-1}$ for all $d \in \mathcal{D}(R)$ and all $j \in \mathbb{Z}$. Moreover, for each $k \in \mathbb{Z}$ and each $d \in \mathcal{D}(R)$, the element $d \Lambda^{k}$, has only on the " $k$ th-diagonal" possibly non-zero entries, i.e.

$$
\left(d \Lambda^{k}\right)_{i j}=\left\{\begin{aligned}
d_{j+k j+k} & \text { if } i=j+k \\
0 & \text { if } i \neq j+k .
\end{aligned}\right.
$$

Therefore we can write each element $A$ in $M_{\mathbb{Z}}(R)$ uniquely as

1.1.1

$$
A=\sum_{k \in \mathbb{Z}} a_{k} \Lambda^{k}, \text { with } a_{k} \in \mathcal{D}(R) .
$$

To the decomposition (1.1.1) we link some notations: if $A$ is as in (1.1.1) then we write

$$
A_{+}=\sum_{k \geq 0} a_{k} \Lambda^{k} \text { and } A_{-}=\sum_{k<0} a_{k} \Lambda^{k} .
$$

Inside $M_{\mathbb{Z}}(R)$ we consider 2 subspaces that form an algebra w.r.t. the product.

1.1.3 Definition. An element $A \in M_{\mathbb{Z}}(R)$ is called uppertriangular of level $k$, if it can be written as

$$
A=\sum_{l \geq k} a_{l} \Lambda^{l}, \text { with } a_{l} \in \mathcal{D}(R)
$$

The collection of all these elements we denote by $U T_{k}$. It is a direct verification that $U T:=\bigcup_{k \in \mathbb{Z}} U T_{k}$ is an algebra with the product introduced above and that an element of $U T$ is invertible if and only if the leading diagonalcomponent is invertible. Likewise one can introduce

1.1.4 Definition An element $A \in M_{\mathbb{Z}}(R)$ is called lowertriangular of level $k$, if it can be written as

$$
A=\sum_{l \leq k} a_{l} \Lambda^{l}, \text { with } a_{l} \in \mathcal{D}(R) .
$$

The collection of all these elements we denote by $L T_{k}$. If we put again $L T:=\bigcup_{k \in \mathbb{Z}} L T_{k}$, then $L T$ is an algebra and an element is invertible in $L T$ if and only if its leading diagonal part is invertible. 
1.1.5 Remark The product of an element in $L T$ and an element in $U T$ is in general not defined and requires some convergence conditions on both factors in order to make sense.

1.2 The hierarchy we are interested in can conveniently be formulated in terms of a matrix $\hat{L}$ in $L T_{1}$ and a matrix $\tilde{M}$ in $U T_{-1}$ of the form

$$
\tilde{L}=\Lambda+\sum_{j \geq 0} \tilde{l} j \Lambda^{-j} \text { and } \tilde{M}=\sum_{i \geq-1} \tilde{m}_{i} \Lambda^{i} \text { with } \tilde{m}_{-1} \text { invertible. }
$$

For all relevant $j$ and $s$, we will write $\tilde{l}_{j}(s)$ and $\tilde{m}_{j}(s)$ instead of $\left(\tilde{l}_{j}\right)_{s s}$ and $\left(\tilde{m}_{j}\right)_{s s .}$ To a pair $(\tilde{L}, \tilde{M})$ as in 1.2 .1 we associate for all $n \geq 1$ the elements $B_{n}$ and $C_{n}$ in $U T \cap L T$ defined by

$$
B_{n}=\left(\tilde{L}^{n}\right)_{+} \text {and } C_{n}=\left(\tilde{M}^{n}\right)_{-} .
$$

The equations of the hierarchy will consist of a system of differential difference equations for the elements $\left\{\tilde{l}_{j}(s), \tilde{m}_{i}(s)\right\}$. In order to give the algebraic description of the hierarchy, we consider the $\left\{\tilde{l}_{j}(s), \tilde{m}_{i}(s)\right\}$ first as free commuting variables and we take for $R$ the algebra $B=\mathbb{C}\left[\mathbb{Z}_{J}(\sim), \mathbb{Z}_{\mathcal{I}}(\sim), \mathbb{Z}_{-\nVdash}(\sim)^{-\nVdash}\right]$, where $j \in \mathbb{N}, \beth \in \mathbb{Z}, \beth \geq-\nVdash$, and $s \in \mathbb{Z}$. Clearly, one can define a $\mathbb{C}$-linear derivation of $B$ by simply prescribing the image of all the $\tilde{l}_{j}(s)$ and all the $\tilde{m}_{i}(s)$. In $M_{\mathbb{Z}}(B)$ the following commutators exist and belong to the indicated subsets

$$
\begin{gathered}
{\left[B_{n}, \tilde{L}\right]=\left[-(\tilde{L})_{-}^{n}, \tilde{L}\right] \in L T_{0}} \\
{\left[C_{n}, \tilde{M}\right]=\left[-\left(\tilde{M}^{n}\right)_{+}, \tilde{M}\right] \in U T_{-1}} \\
{\left[B_{n}, \tilde{M}\right] \in U T_{-1} \text { and }\left[C_{n}, \tilde{L}\right] \in L T_{0}}
\end{gathered}
$$

In the light of the foregoing remark, we can define $\mathbb{C}$-linear derivations $\tilde{\partial}_{t_{n}}$ and $\tilde{\partial}_{s_{n}}$ of $B$ by the equations

$$
\tilde{\partial}_{t_{n}} \tilde{L}=\left[B_{n}, \tilde{L}\right], \tilde{\partial}_{t_{n}} \tilde{M}=\left[B_{n}, \tilde{M}\right]
$$

$$
\tilde{\partial}_{s_{n}} \tilde{L}=\left[C_{n}, \tilde{L}\right], \tilde{\partial}_{s_{n}} \tilde{M}=\left[C_{n}, \tilde{M}\right]
$$

Let $R$ be a general $\mathbb{C}$-algebra and let $(L, M)$ be a pair of elements in $M_{\mathbb{Z}}(R)$ of the form 1.2.1. These data are the same as giving a $C$-algebra homomorphism $\alpha: B \rightarrow R$ by the prescription

$$
\alpha\left(\tilde{l}_{j}(s)\right)=\left(l_{j}\right)_{s s} \text { and } \alpha\left(\tilde{m}_{z}(s)\right)=\left(m_{\imath}\right)_{s s} .
$$


The map $\alpha$ defines by coefficientwise action a $\mathbb{C}$-linear map $\alpha: M_{\mathbb{Z}}(B) \rightarrow M_{\mathbb{Z}}(R)$ and we have $L=\alpha(\tilde{L})$ and $M=\alpha(\tilde{M})$. Now we would like to have derivations $\partial_{t_{n}}$ and $\partial_{s_{n}}, n \geq 1$, of $R$ that are prolongations of the derivations $\tilde{\partial}_{t_{n}}$ and $\tilde{\partial}_{s_{n}}$ defined by 1.2 .2 and 1.2 .3 , i.e. they should satisfy for all $n \geq 1$,

$$
\alpha \circ \tilde{\partial}_{t_{n}}=\partial_{t_{n}} \circ \alpha \text { and } \alpha \circ \tilde{\partial}_{s_{n}}=\partial_{s_{n}} \circ \alpha
$$

One directly verifies that condition 1.2 .5 is equivalent to showing that the pair $(L, M)$ and the derivations $\left\{\partial_{t_{n}}, \partial_{s_{n}}, n \geq 1\right\}$ satisfy the equations

$$
\partial_{t_{n}} L=\left[(L)_{+}^{n}, L\right], \partial_{t_{n}} M=\left[\left(L^{n}\right)_{+}, M\right]
$$

$$
\partial_{s_{n}} M=\left[(M)_{-}^{n}, M\right] \text { and } \partial_{s_{n}} L=\left[(M)_{-}^{n}, L\right] .
$$

The equations 1.2.6 and 1.2.7 are called the equations of the Toda lattice hierarchy, since the simplest non-linear equations contained in it are that of the generalized Toda lattice, see [UT]. The data $\left(R, \partial_{t_{n}}, \partial_{s_{n}}, \alpha(\tilde{L}), \alpha(\tilde{M})\right)$ we call a solution of this hierarchy.

¿From now on, we assume that $R$ is a $\mathbb{C}$-algebra of functions in the parameters $\left\{t_{n} \mid n \geq 1\right\}$ and $\left\{s_{n} \mid n \geq 1\right\}$, that it is stable under taking the partial derivative $\partial_{t_{n}}$ resp. $\partial_{s_{n}}$ w.r.t. $t_{n}$ resp. $s_{n}$, and that $R$ contains $\mathbb{C}\left[\approx_{\propto}, \sim_{\alpha}, \ltimes \geq \nVdash\right]$. We will now comment on the linear problem associated to $(1.2 .6)$ and $(1.2 .7)$ in [UT]. There they considered "operators" $W^{(\infty)}$ and $W^{(0)}$ of the form

$$
\begin{array}{r}
W^{(\infty)}=\widehat{W}^{(\infty)} \cdot \exp \left(\sum_{i>0} t_{i} \Lambda^{i}\right):=\left\{\mathrm{Id}+\sum_{i<0} \omega_{i}^{(\infty)} \Lambda^{i}\right\} \exp \left(\sum_{i>0} t_{i} \Lambda^{i}\right) \text { and } \\
W^{(0)}=\widehat{W}^{(0)} \cdot \exp \left(\sum_{i>0} s_{i} \Lambda^{-i}\right):=\left\{\sum_{i \geq 0} \omega_{i}^{(0)} \Lambda^{i}\right\} \exp \left(\sum_{i>0} s_{i} \Lambda^{-i}\right), \text { with } \omega_{0}^{(0)} \text { invertible, }
\end{array}
$$

where all the $\omega_{i}^{(\infty)}$ and $\omega_{i}^{(\circ)}$ belong to $\mathcal{D}(R)$ and such that $\omega_{0}^{\left({ }^{\circ}\right)}$ is invertible in $\mathcal{D}(R)$.

Since $W^{(\infty)}$ and $W^{(0)}$ are products of an element in $U T$ and in $L T$, these products make in general no sense in $M_{\mathbb{Z}}(R)$ and one must give some convergent context such that $W^{(\infty)}$ and $W^{(0)}$ can be seen as elements of $M_{Z}(R)$. This will be done in the second and third section. In that case one notes that both $W^{(\infty)}$ and $W^{(0)}$ are invertible in $M_{\mathbb{Z}}(R)$.

If $W^{\infty}$ and $W^{0}$ are well-defined elements of $M_{\mathbb{Z}}(R)$, then we will call them wavematrices in $M_{\mathbb{Z}}(R)$. The linear system associated with $(L, M)$ consists of the following equations that couple the wavematrices to the pair $(L, M)$,

$$
L W^{(\infty)}=W^{(\infty)} \Lambda \text { and } M W^{(0)}=W^{(0)} \Lambda^{-1}
$$




$$
\partial_{s_{n}} W^{(\infty)}=C_{n} W^{(\infty)} \text { and } \partial_{s_{n}} W^{(0)}=C_{n} W^{(0)} \text { for all } n \geq 1
$$

Since $W^{(\infty)}$ and $W^{(0)}$ are invertible, equation (1.2.8) lead to

$$
L=\widehat{W}^{(\infty)} \Lambda \widehat{W}^{(\infty)^{-1}} \text { and } M=\widehat{W}^{(0)} \Lambda^{-1} \widehat{W}^{(0)^{-1}}
$$

By differentiating the equations in (1.2.8) w.r.t. the variable $t_{n}$ resp. $s_{n}$ and by substituting (1.2.9) resp. (1.2.10), we get that $L$ and $M$ defined by (1.2.11) satisfy the equations (1.2.3). In the rest of this paper we present a geometric context from which one can construct wave matrices $W^{(\infty)}$ and $W^{(0)}$ in $M_{\mathbb{Z}}(R)$ that satisfy (1.2.9) and (1.2.10). The operators $L$ and $M$ defined by (1.2.11) are then the solutions of the Toda lattice hierarchy.

\section{$\S 2$ The geometric setting.}

2.1 Let $H$ be a complex Hilbert space with orthonormal basis $\left\{e_{i} \mid i \in \mathbb{Z}\right\}$ and innerproduct $\langle\cdot\rangle$. The space of bounded linear operators from $H$ to $H$, we denote by $B(H)$ and we assume it to be equiped with the operator norm. Its group of invertible elements is denoted by $G l(H)$. The group $G l(H)$ is an open part of $B(H)$ and as such, it is a Banach Lie group with Lie algebra $B(H)$.

2.1.1 Notation To each operator $g$ in $B(H)$ we associate a $\mathbb{Z} \times \mathbb{Z}$-matrix $[g]=\left([g]_{i j}\right)$ in $M_{\mathbb{Z}}(\mathbb{C})$ by putting $[g]_{i j}=<g\left(e_{j}\right)\left|e_{i}\right\rangle, i, j \in \mathbb{Z}$.

Next we introduce some Lie subgroups of $G l(H)$ and their corresponding Lie algebras. First of all we have the Borel subgroup $B_{+}$and the "opposite" Borel subgroup $B_{-}$given by

$$
\begin{aligned}
& B_{+}=\left\{g \mid g \in G l(H),[g]_{i j}=0=\left[g^{-1}\right]_{i j} \text { for all } i<j\right\}, \\
& B_{-}=\left\{g \mid g \in G l(H),[g]_{i j}=0=\left[g^{-1}\right]_{i j} \text { for all } i>j\right\} .
\end{aligned}
$$

Their Lie algebras $L\left(B_{+}\right)$and $L\left(B_{-}\right)$satisfy

$$
\begin{gathered}
L\left(B_{+}\right)=\left\{b \mid b \in B(H),[b]_{i j}=0 \text { for all } i<j\right\} \\
L\left(B_{-}\right)=\left\{b \mid b \in B(H),[b]_{i j}=0 \text { for all } i>j\right\} .
\end{gathered}
$$

As in the finite-dimensional case, $B_{+}$and $B_{-}$are the semi-direct product of a diagonal group

$$
D=\left\{g \mid g \in G l(H),[g]_{i j}=0 \text { for all } i \neq j\right\}
$$

and the unipotent subgroups $U_{+}$respectively $U_{-}$given by 


$$
U_{+}=\left\{g \mid g \in B_{+},[g]_{i i}=1 \text { for all } i \in \mathbb{Z}\right\} \text { and } \mathbb{U}_{-}=\left\{\tilde{\partial} \mid \widetilde{\partial} \in \mathbb{B}_{-},[\tilde{\sigma}]_{\beth ב}=\nVdash \text { for all } \beth \in \mathbb{Z}\right\} \text {. }
$$

Their Lie algebras are denoted respectively by $L(D), L\left(U_{+}\right)$and $L\left(U_{-}\right)$. Since $B(H)$ decomposes as

$$
B(H)=L\left(U_{-}\right) \oplus L(D) \oplus L\left(U_{+}\right)
$$

and exp is a local diffeomorphism around zero, we see that

$$
O_{1}=B_{+} U_{-} \quad \text { and } O_{2}=U_{-} B_{+}
$$

are open subsets of $G l(H)$. We will give another characterization of $O_{1}$ and $O_{2}$. For each $n \in \mathbb{Z}$, let $H_{n}$ be the topological span of the $\left\{e_{i} \mid i \geq n\right\}$ and let $p_{n}$ be the orthogonal projection onto $H_{n}$. If one decomposes an operator $g \in O_{1}$ and $h \in O_{2}$ w.r.t. $H=H_{n} \oplus H_{n}^{\perp}$, then one computes directly that for all $n \in \mathbb{Z}$

$$
g=\left(\begin{array}{ll}
g_{11}(n) & g_{12}(n) \\
g_{21}(n) & g_{22}(n)
\end{array}\right) \text { with } g_{22}(n) \in G l\left(H_{n}^{\perp}\right) \text { and }
$$

$$
h=\left(\begin{array}{ll}
h_{11}(n) & h_{12}(n) \\
h_{21}(n) & h_{22}(n)
\end{array}\right) \text { with } h_{11}(n) \in G l\left(H_{n}\right) .
$$

Reversely, these properties characterize the sets $O_{1}$ and $O_{2}$, for we have

2.1.4 Proposition The sets $O_{1}$ resp. $O_{2}$ consist of all $g$ resp. $h$ in $G l(H)$ satisfying (2.1.2) resp. (2.1.3) for all $n \in \mathbb{Z}$.

Proof We give the proof for $O_{2}$, the one for $O_{1}$ is similar. Take any $n \in \mathbb{Z}$ then one has

$$
h=\left(\begin{array}{cc}
\operatorname{Id} & 0 \\
h_{21}(n) h_{11}(n)^{-1} & \text { Id }
\end{array}\right)\left(\begin{array}{cc}
h_{11}(n) & h_{12}(n) \\
0 & h_{22}(n)-h_{21}(n) h_{11}(n)^{-1} h_{12}(n)
\end{array}\right) .
$$

Hence we may assume $h_{21}(n)=0$. With respect to the decomposition $H_{n}=<e_{n}>\oplus H_{n+1}$ we have that

$$
h_{11}(n)=\left(\begin{array}{cc}
h_{11}(n+1) & \beta \\
\gamma & \delta
\end{array}\right)=\left(\begin{array}{cc}
\operatorname{Id} & 0 \\
\gamma h_{11}(n+1)^{-1} 1 & \text { Id }
\end{array}\right)\left(\begin{array}{cc}
h_{11}(n+1) \\
0
\end{array}\right) .
$$

Continuing in this fashion we can find an $u_{1}$ in $G l\left(H_{n}\right)$ and a $b_{1}$ in $G l\left(H_{n}\right)$ such that $h_{11}(n)=$ $u_{1} b_{1}$ and their matrices w.r.t. the $\left\{e_{k} \mid k \geq n\right\}$ have the form

$$
\left[u_{1}\right]=\left(\begin{array}{cccc}
\ddots & & & 0 \\
& 1 & & \\
& & \ddots & \\
* & & & 1
\end{array}\right) \text { and }\left[b_{1}\right]=\left(\begin{array}{ccccc}
\ddots & \ddots & & & * \\
& 0 & * & & \\
& & \ddots & \ddots & \\
\cdots & 0 & \cdots & 0 & *
\end{array}\right) \text {. }
$$


Since $h$ belongs to $O_{2}$ and since we may assume $h_{21}(n)=0$, one sees that $h_{22}(n)$ decomposes w.r.t. $H_{n}^{\perp}=<e_{n-1}>\oplus H_{n-1}^{\perp}$ as

$$
h_{22}(n)=\left(\begin{array}{cc}
\alpha_{1} & \beta_{1} \\
\gamma_{1} & h_{22}(n-1)
\end{array}\right) \text { with } \alpha_{1} \neq 0
$$

Hence we can solve by a step by step procedure that there is a $u_{2}$ and a $b_{2}$ in $G l\left(H_{n}^{\perp}\right)$ such that $h_{22}(n)=u_{2} b_{2}$ and such that their matrices w.r.t. the $\left\{e_{k} \mid k<n\right\}$ have the form

$$
\left[u_{2}\right]=\left(\begin{array}{ccccc}
1 & & & & 0 \\
* & \ddots & & & \\
\vdots & \ddots & 1 & & , \\
& & * & \ddots & \\
& & & \ddots & \ddots
\end{array}\right) \text { and }\left[b_{2}\right]=\left(\begin{array}{cccc}
* & \cdots & * & \cdots \\
0 & \ddots & & \\
\vdots & \ddots & * & \\
0 & & 0 & \ddots \\
& & & \ddots
\end{array}\right) .
$$

By combining the $u_{1}, u_{2}, b_{1}, b_{2}$ and $h_{12}(n)$ and $h_{21}(n)$, one finds the desired decomposition of $h$. This concludes the proof of the proposition.

2.2 Next we introduce the flows that play a role in the Toda lattice hierarchy. For each open neighbourhood $U$ of $\{\lambda|\lambda \in \mathbb{C},| \lambda \mid=\nVdash\}$, consider

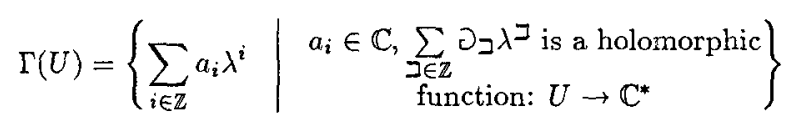

with the topology of uniform convergence on compact subsets. It is a group w.r.t. point wise multiplication. Let $\tilde{\Gamma}$ be the direct limit of the $\Gamma(U)$ with the corresponding topology. Inside $\tilde{\Gamma}$ we have the subgroups $\tilde{\Gamma}_{+}$and $\tilde{\Gamma}_{-}$given by

$$
\tilde{\Gamma}_{+}=\left\{\exp \left(\sum_{i>0} t_{i} \lambda^{i}\right) \in \tilde{\Gamma}\right\} \text { and } \tilde{\Gamma}_{-}=\left\{\exp \left(\sum_{j>0} s_{j} \lambda^{-j}\right) \in \tilde{\Gamma}\right\}
$$

According to [HP], each element $\gamma$ in $\tilde{\Gamma}$ can be decomposed uniquely as follows

$$
\gamma=\gamma_{+} \gamma_{-} \lambda^{k} a, \text { with } \gamma_{+} \in \tilde{\Gamma}_{+}, \gamma_{-} \in \tilde{\Gamma}_{-}, k \in \mathbb{Z} \text { and } \partial \in \mathbb{C}^{*} .
$$

Let $\tilde{\Gamma}(0)$ be the subgroup of $\tilde{\Gamma}$ consisting of all $\gamma$ in $\tilde{\Gamma}$ with $k$ equal to zero. The group $\tilde{\Gamma}$ maps continuously into $G l(H)$. For, let $\underline{\Lambda}: H \rightarrow H$ be the shift operator defined by

$$
\underline{\Lambda}\left(\sum_{i} \alpha_{i} e_{i}\right)=\sum \alpha_{i} e_{i+1}
$$

Then we define a continuous embedding $M: \tilde{\Gamma} \rightarrow G l(H)$ by

$$
M(\gamma)=M\left(\sum_{i \in \mathbb{Z}} a_{i} \lambda^{i}\right)=\sum_{i \in \mathbb{Z}} a_{i} \underline{\Lambda}^{i} .
$$


The image under $M$ of $\tilde{\Gamma}, \tilde{\Gamma}_{+}, \tilde{\Gamma}_{-}$and $\tilde{\Gamma}(0)$, we denote respectively by $\Gamma, \Gamma_{+}, \Gamma_{-}$and $\Gamma(0)$. Note that $\Gamma(0)$ corresponds exactly with the intersection of $\Gamma$ with the open sets $O_{1}$ and $O_{2}$.

2.2.2 Remark Since the $\mathbb{Z} \times \mathbb{Z}$-matrix of $\Lambda$ is exactly the matrix $\Lambda$ from section (1.1), it will be clear that the matrices of elements of $\Gamma_{+}$and $\Gamma_{-}$are exactly the matrices occurring in the wavematrices of section (1.2).

\section{$\S 3$ The construction of the solutions.}

3.1 Inside $G l(H)$ we consider the open subset $\Omega$ defined by

$$
\Omega=\Gamma(0) O_{2} \Gamma(0) .
$$

Since $\Gamma(0)$ is the union of the $a \Gamma_{+} \Gamma_{-}$, with $a \in \mathbb{C}^{*}$, and since the open set $O_{2}$ can be written as $U_{-} B_{+}$, we see that

$$
\Omega=\Gamma_{+} O_{2} \Gamma_{-} .
$$

The set $\Omega$ is not equal to $G l(H)$, for if one considers for example the orbit $\Gamma_{+} \Lambda^{k} \Gamma_{-}$, with $k \neq 0$, then it has empty intersection with $O_{2}$. For, each $g$ in $\Gamma_{+} \Lambda^{k} \Gamma_{-}$decomposes w.r.t. $H=H_{n} \oplus H_{n}^{\perp}$ as

$$
g=\left(\begin{array}{ll}
g_{11} & g_{12} \\
g_{21} & g_{22}
\end{array}\right), \text { with } g_{11} \text { a Fredholm operator of index }-k .
$$

Hence, by lemma (2.1.4) these elements do not belong to $O_{2}$. Now we take elements $\gamma_{+}$in $\Gamma_{+}$and $\gamma_{-}$in $\Gamma_{-}$such that

$$
\gamma_{+}=\gamma_{+}(t)=\exp \left(\sum_{i>0} t_{i} \underline{\Lambda}^{i}\right) \text { and } \gamma_{-}=\gamma_{-}(s)=\exp \left(\sum_{j>0} s_{j} \underline{\Lambda}^{-j}\right),
$$

and we consider the left $\Gamma_{+}$-flow and the right $\Gamma_{-}$-flow in $\Omega$. That is to say, we choose a $g$ in $\Omega$ and we look at

$$
G(t, s)=\gamma_{+} g \gamma_{-}^{-1} .
$$

Clearly $G$ is a holomorphic map from $\tilde{\Gamma}_{+} \times \tilde{\Gamma}_{-}$to $G l(H)$ and since $g$ belongs to $\Omega$, we have that $G^{-1}\left(O_{2}\right)$ is a non-empty open subset of $\tilde{\Gamma}_{+} \times \tilde{\Gamma}_{-}$. For the ring $R$ in the first section we take now the ring of holomorphic functions on $G^{-1}\left(O_{2}\right)$. For all $\left(\gamma_{+}(t), \gamma_{-}(s)\right)$ in $G^{-1}\left(O_{2}\right)$, we have a decomposition

$$
G(t, s)=\widehat{\mathcal{W}}^{(\infty)}(t, s)^{-1} \widehat{\mathcal{W}}^{(0)}(t, s),
$$

with $\widehat{\mathcal{W}}^{(\infty)}(t, s) \in U_{-}$and $\widehat{\mathcal{W}}^{(0)}(t, s) \in B_{+}$. If we write $\widehat{W}^{(\infty)}$ resp. $\widehat{W}^{(0)}$ for the matrices of $\widehat{W}^{(\infty)}$ resp. $\widehat{W}^{(0)}$, then the coefficients of these matrices belong to $R$, and they decompose as 


$$
\begin{aligned}
& \widehat{W}^{(\infty)}=\Lambda^{0}+\sum \underline{\omega}_{j}^{(\infty)} \Lambda^{j}, \text { with } \underline{\omega}_{j}^{(\infty)} \in \mathcal{D}(R) \text { and } \\
& \widehat{W}^{(0)}=\sum_{i \geq 0} \underline{\omega}_{i}^{(0)} \Lambda^{i}, \text { with } \underline{\omega}_{i}^{(0)} \in \mathcal{D}(R) \text { and } \underline{\omega}_{0}^{(0)} \text { invertible in } \mathcal{D}(R)
\end{aligned}
$$

Next we introduce the operators $\mathcal{W}^{(\infty)}$ and $\mathcal{W}^{(0)}$ in $G l(H)$ by

$$
\mathcal{W}^{(\infty)}=\widehat{\mathcal{W}}^{(\infty)} \cdot \gamma_{+} \text {and } \mathcal{W}^{(0)}=\widehat{\mathcal{W}}^{(0)} \cdot \gamma_{-} .
$$

Denote the matrices of $\mathcal{W}^{(\infty)}$ and $\mathcal{W}^{(0)}$ by $W^{(\infty)}$ resp. $W^{(0)}$. The set-up has been chosen such that in $M_{\mathbb{Z}}(R)$ the products of $\left.\widehat{W}^{\infty}\right)$ and $\left[\gamma_{+}\right]$and of $\widehat{W}^{(0)}$ and $\left[\gamma_{-}\right]$are well-defined and that they are equal to $W^{(\infty)}$ resp. $W^{(0)}$. Again the coefficients of $W^{(\infty)}$ and $W^{(0)}$ belong to $R$. Moreover they have the form of a "wavematrix" as considered in section (1.2).

To $W^{(\infty)}$ and $W^{(0)}$ we associate the Lax-matrices $L$ and $M$ according to

$$
\text { 3.1.2 } L_{g}=W^{(\infty)} \Lambda W^{(\infty)^{-1}}=\widehat{W}^{(\infty)} \Lambda \widehat{W}^{(\infty)^{-1}} \text { and } M_{g}=W^{(0)} \Lambda W^{(0)^{-1}}=\widehat{W}^{(0)} \Lambda \widehat{W}^{(0)^{-1}} \text {. }
$$

We are now ready to prove the main result.

3.1.3 Theorem (a) If $g$ belongs to $\Omega$, then the matrices $L_{g}$ and $M_{g}$ constructed in (3.1.2) are solutions of the Toda lattice hierarchy.

(b) If $\delta_{-} \in \Gamma_{-}, \delta_{+} \in \Gamma_{+}$and $a \in \mathbb{C}^{*}$ then the constructed solutions of the Toda lattice hierarchy corresponding to $g$ and $\delta_{-} g a \delta_{+}$are the same, i.e.

$$
L_{\delta_{-} g a \delta_{+}}=L_{g} \text { and } M_{\delta_{-} g a \delta_{+}}=M_{g} .
$$

Proof First of all we note that $\mathcal{W}^{(0)}$ and $\mathcal{W}^{(\infty)}$ are constructed in such a way that $W^{(\infty)}[g]=$ $W^{(0)}$. Hence, if we can proof

$$
\partial_{t_{n}} W^{(\infty)}=B_{n} W^{(\infty)} \text { for all } n \geq 1,
$$

then the same equations holds for $W^{(0)}$. Analogously, it suffices to prove

$$
\partial_{s_{n}} W^{(0)}=C_{n} W^{(0)} \text { for all } n \geq 1,
$$

to get these equations for $W^{(\infty)}$. Consider first the equations (1.2.9). On one hand we have

$$
\begin{aligned}
\partial_{t_{n}} W^{(\infty)} & =\partial_{t_{n}}\left(\widehat{W}^{(\infty)}\right) \exp \left(\sum_{i>0} t_{i} \Lambda^{i}\right)+\widehat{W}^{(\infty)} \Lambda^{n} \exp \left(\sum_{i>0} t_{i} \Lambda^{i}\right) \\
& =\left\{\partial_{t_{n}}\left(\widehat{W}^{(\infty)}\right)+\widehat{W}^{(\infty)} \Lambda^{n}\right\} \widehat{W}^{(\infty)^{-1}} W^{(\infty)} \\
& =\left\{\partial_{t_{n}}\left(\widehat{W}^{(\infty)}\right) \widehat{W}^{(\infty)^{-1}}+L^{n}\right\} W^{(\infty)} .
\end{aligned}
$$

On the other hand, if we differentiate $W^{(0)}[g]^{-1}$, w.r.t. $t_{n}$, we get 


$$
\begin{aligned}
\partial_{t_{n}} W^{(0)}[g]^{-1} & =\partial_{t_{n}}\left(\widehat{W}^{(0)}\right) \exp \left(\sum_{j>0} s_{j} \Lambda^{-j}\right)[g]^{-1} \\
& =\left\{\partial_{t_{n}}\left(\widehat{W}^{(0)}\right) \widehat{W}^{(0)^{-1}}\right\} \widehat{W}^{(\infty)} \\
& =\left\{\sum_{k \geq 0} b_{k} \Lambda^{k}\right\} \widehat{W}^{(\infty)}
\end{aligned}
$$

In particular we may conclude that

$$
\sum_{k \geq 0} b_{k} \Lambda^{k}=\left\{\partial_{t_{n}}\left(\widehat{W}^{(\infty)}\right) \widehat{W}^{(\infty)^{-1}}+L^{n}\right\}_{+}=\left(L^{n}\right)_{+}=B_{n} .
$$

The second equality in this equation follows from the fact that $\partial_{t_{n}}\left(\widehat{W}^{(\infty)}\right)$ is lowertriangular of level -1 . The equations (1.2.10) are also obtained by differentiating once $W^{(0)}$ and once $W^{(\infty)}[g]$ :

$$
\begin{aligned}
\partial_{s_{n}}\left(W^{(0)}\right) & =\left\{\partial_{s_{n}}\left(\widehat{W}^{(0)}\right)+\widehat{W}^{(0)} \Lambda^{-1}\right\} \exp \left(\sum_{j>0} s_{j} \Lambda^{-j}\right) \\
& =\left\{\partial_{s_{n}}\left(\widehat{W}^{(0)}\right) \widehat{W}^{(0)^{-1}}+M^{n}\right\} W^{(0)} \\
\partial_{s_{n}}\left(\widehat{W}^{(\infty)}[g]\right) & =\partial_{s_{n}}\left(\widehat{W}^{(\infty)}\right) \exp \left(\sum_{i>0} t_{i} \Lambda^{i}\right) \\
& =\left\{\partial_{s_{n}}\left(\widehat{W}^{(\infty)}\right) \widehat{W}^{(\infty)^{-1}}\right\} \widehat{W}^{(\infty)}[g] .
\end{aligned}
$$

Since $\partial_{s_{n}}\left(\widehat{W}^{(\infty)}\right)$ is lowertriangular of level -1 and $\partial_{s_{n}}\left(\widehat{W}^{(0)}\right)$ is uppertriangular of level 0 , we get that

$$
\left\{\partial_{s_{n}}\left(\widehat{W}^{(\infty)}\right) \widehat{W}^{(\infty)^{-1}}\right\}=\left\{\partial_{s_{n}}\left(\widehat{W}^{(0)}\right) \widehat{W}^{(0)^{-1}}+M^{n}\right\}_{-}=\left(M^{n}\right)_{-}=C_{n} .
$$

This proves the equations (1.2.9) and (1.2.10) for $L_{g}$ and $M_{g}$ and the first part of the theorem. Next we consider $\tilde{g}=\delta_{-} g a \delta_{+}$, with $a \in \mathbb{C}^{*}, \delta_{-} \in \bigwedge_{-}, \delta_{+} \in \varliminf_{+}$and $g \in \Omega$. It belongs again to $\Omega$ and, since $\delta_{-}, \gamma_{+}, \delta_{+}$and $\gamma_{-}$commute the corresponding wavematrices are easily seen to have the form $W^{(\infty)}\left[\delta_{-}\right]^{-1}$ and $W^{(0)}\left[\delta_{+}\right] a$, where $W^{(\infty)}$ and $W^{(0)}$ are the wavematrices belonging to $g$. This implies that $L_{\tilde{g}}$ and $M_{\bar{g}}$ are given by

$$
\begin{aligned}
L_{\tilde{g}} & =\widehat{W}^{(\infty)}\left[\partial_{-}\right]^{-1} \Lambda\left[\partial_{-}\right] \widehat{W}^{(\infty)^{-1}}=L_{g} \text { and } \\
M_{\tilde{g}} & =\widehat{W}^{(0)}\left[\partial_{+}\right] a \Lambda^{-1} a^{-1}\left[\partial_{+}\right]^{-1} \widehat{W}^{(0)^{-1}}=\widehat{W}^{(0)} \Lambda \widehat{W}^{(0)^{-1}}=M_{g}
\end{aligned}
$$

This concludes the proof of the theorem. 


\section{References}

[UT] K. Ueno and K. Takasaki, Toda Lattice Hierarchy, Publication 423, RIMS Kyoto 1983.

[PS] A. Pressley and G. Segal, Loop groups, Oxford Mathematical Monographs, Clarendon Press, Oxford, 1986.

[HP] G.F. Helminck and G.F. Post, The geometry of differential difference equations, Memorandum 999, University of Twente, september 1991. 